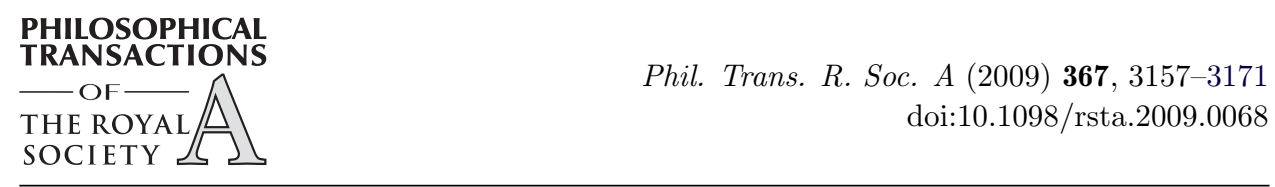

\title{
Entropic transport: a test bed for the Fick-Jacobs approximation
}

\author{
By P. Sekhar Burada*, Gerhard Schmid and Peter Hänggi \\ Institut für Physik, Universität Augsburg, Universitätsstrasse 1, D-86135 \\ Augsburg, Germany
}

\begin{abstract}
Biased diffusive transport of Brownian particles through irregularly shaped, narrow confining quasi-one-dimensional structures is investigated. The complexity of the higher dimensional diffusive dynamics is reduced by means of the so-called Fick-Jacobs approximation, yielding an effective one-dimensional stochastic dynamics. Accordingly, the elimination of transverse, equilibrated degrees of freedom stemming from geometrical confinements and/or bottlenecks causes entropic potential barriers that the particles have to overcome when moving forward noisily. The applicability and the validity of the reduced kinetic description are tested by comparing the approximation with Brownian dynamics simulations in full configuration space. This non-equilibrium transport in such quasi-one-dimensional irregular structures implies, for moderate-to-strong bias, a characteristic violation of the Sutherland-Einstein fluctuation-dissipation relation.
\end{abstract}

Keywords: Brownian motion; entropic transport; Fick-Jacobs equation

\section{Introduction}

Diffusion of Brownian particles through narrow, tortuous confining structures such as micropores and nanopores, zeolites, biological cells and microfluidic devices plays a prominent role in the dynamical characterization of these systems (Barrer 1978; Volkmuth \& Austin 1992; Liu et al. 1999; Kettner et al. 2000; Müller et al. 2000; Hille 2001; Nixon \& Slater 2002; Matthias \& Müller 2003; Berezhkovskii \& Bezrukov 2005; Siwy et al. 2005). Effective control schemes for transport in these systems require a detailed understanding of the diffusive mechanisms involving small objects and, in this regard, an operative measure to gauge the role of fluctuations. The study of these transport phenomena is, in many respects, equivalent to an investigation of geometrically constrained Brownian dynamics (Mazo 2002; Burada et al. 2009; Hänggi \& Marchesoni 2009). With this work, we focus on the stochastic transport of small-sized particles in confined geometries and the feasibility of the so-called Fick-Jacobs (FJ) approximation to describe the steady-state particle densities. Restricting the volume of the configuration space available to the diffusing particles by means of confining boundaries or obstacles discloses intriguing entropic phenomena (Liu et al. 1999).

*Author for correspondence (sekhar.burada@physik.uni-augsburg.de).

One contribution of 14 to a Theme Issue 'Topics on non-equilibrium statistical mechanics and nonlinear physics'. 
The driven transport of charged particles across bottlenecks (Burada et al. 2009), such as ion transport through artificial nanopores or artificial ion pumps (Siwy et al. 2005; Kosinska et al. 2008) or in biological channels (Berezhkovskii \& Bezrukov 2005), are more familiar systems where diffusive transport is regulated by entropic barriers. Similarly, the operation of artificial Brownian motors and molecular machines also relies on a mutual interplay among diffusion and binding action by energetic or, more relevant in the present context, entropic barriers (Derenyi \& Astumian 1998; Astumian \& Hänggi 2002; Reimann \& Hänggi 2002; Burada et al. 2009; Hänggi \& Marchesoni 2009).

The outline of this work is as follows: in $\S 2$, we introduce our model and formulate the mathematical formalism needed to model the diffusion of a Brownian particle immersed in a confined medium. In $\S 3$, we present the FJ approximation and compute the entropic effects on the particle transport and on the steady-state probability density in the presence of an applied force in the transport direction. In $\S 4$, we compare the precise two-dimensional numerical simulation results with those obtained from applying the FJ approximation. In $\S 5$, we discuss the effective lateral diffusion and test the Sutherland-Einstein fluctuation-dissipation relation. Section 6 provides a discussion of our main findings.

\section{Overdamped system dynamics}

Generic mass transport through confined structures such as irregular pores and channels, cf. the one depicted in figure 1, is governed by the transport of suspended Brownian particles subjected to an externally applied potential $V(\boldsymbol{r})$. Generally, the dynamics of the Brownian particle inside the medium can be well described by the Langevin dynamics in the over-damped limit (Purcell 1977), with reflecting boundary conditions at the channel walls. The stochastic dynamics then reads

$$
\eta \dot{\boldsymbol{r}}(\tilde{t})=-\nabla V(\boldsymbol{r}(\tilde{t}))+\sqrt{\eta k_{\mathrm{B}} T} \boldsymbol{\xi}(\tilde{t}),
$$

where $r$ is the position vector of a Brownian particle at time $\tilde{t}, \eta$ denotes the friction coefficient, $k_{\mathrm{B}}$ is the Boltzmann constant and $T$ refers to the environmental temperature. Thermal fluctuations due to the coupling of the Brownian particle to the environment are modelled by Gaussian white noise with zero mean and an auto-correlation function obeying the Sutherland-Einstein fluctuation-dissipation relation (Hänggi \& Marchesoni 2005)

$$
\left\langle\xi_{i}(\tilde{t}) \xi_{j}\left(\tilde{t}^{\prime}\right)\right\rangle=2 \delta_{i j} \delta\left(\tilde{t}-\tilde{t}^{\prime}\right), \quad \text { for } \quad i, j=x, y, z .
$$

For simplicity, we consider the dynamics of a Brownian particle that is subjected to a constant force $\boldsymbol{F}=F \boldsymbol{e}_{x}$ acting along the direction of the channel axis (in the $x$-direction here). The Langevin equation for the over-damped dynamics then reads

$$
\eta \dot{\boldsymbol{r}}(\tilde{t})=\boldsymbol{F}+\sqrt{\eta k_{\mathrm{B}} T} \boldsymbol{\xi}(\tilde{t}),
$$

with reflecting (i.e. no across-flow) boundary conditions implied at the channel walls, which confine the Brownian particles within the channel geometry. 


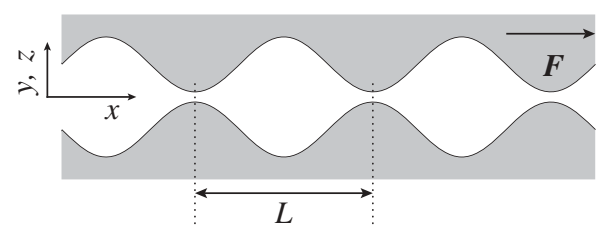

Figure 1. Schematic diagram of a channel confining the motion of laterally forced (with strength $F$ ) Brownian particles. The half-width $\omega$ is a periodic function of $x$ with periodicity $L$.

In order to further simplify the treatment of this setup, we introduce dimensionless variables. We measure all lengths in units of the period length $L$, i.e. $\boldsymbol{r}=L \boldsymbol{x}$, where $\boldsymbol{x}$ denotes the dimensionless position vector of the particle. As the unit of time $\tau$, we choose twice the time the particle takes to diffusively overcome the distance $L$, which is given by $\tau=L^{2} \eta /\left(k_{\mathrm{B}} T\right)$, i.e. $\tilde{t}=\tau t$ (Burada et al. 2008b). In these dimensionless variables, the Langevin dynamics assumes the form

$$
\frac{\mathrm{d} \boldsymbol{x}}{\mathrm{d} t}=\boldsymbol{f}+\boldsymbol{\xi}(t)
$$

where $\langle\xi(t)\rangle=0,\left\langle\xi_{i}(t) \xi_{j}\left(t^{\prime}\right)\right\rangle=2 \delta_{i j} \delta\left(t-t^{\prime}\right)$, for $i, j=x, y, z$, and the dimensionless force becomes

$$
f=f \boldsymbol{e}_{x} \quad \text { and } \quad f=\frac{L F}{k_{\mathrm{B}} T} .
$$

The performed dimensionless scaling parameter $f$ characterizes the force as 'the ratio between the work $L F$ done on the particle along a distance of the period length $L$ and the thermal energy $k_{\mathrm{B}} T^{\prime}$. We anticipate here the fact that, in the case of diffusion occurring in purely energetic potential landscapes, the driving force $F$ and the temperature $T$ are independent variables; in contrast, in systems with entropic features, these two quantities become coupled (Reguera et al. 2006). In order to adjust a certain value of $f$, one can modify either the force strength $F$ or adjust the noise intensity $k_{\mathrm{B}} T$.

The corresponding Fokker-Plank equation describing the time evolution of the probability density $P(\boldsymbol{x}, t)$ takes the form (Hänggi \& Thomas 1982; Risken 1989)

$$
\frac{\partial P(\boldsymbol{x}, t)}{\partial t}=-\nabla \cdot \boldsymbol{J}(\boldsymbol{x}, t),
$$

where $\boldsymbol{J}(\boldsymbol{x}, t)$ is the probability current

$$
\boldsymbol{J}(\boldsymbol{x}, t)=(\boldsymbol{f}-\boldsymbol{\nabla}) P(\boldsymbol{x}, t) .
$$

Note that, for channels with similar geometry which are related by a scale transformation $\boldsymbol{r} \rightarrow \lambda \boldsymbol{r}, \lambda>0$, the transport properties are determined by a single dimensionless parameter $f$ that subsumes the respective period length, the external force and the temperature of the surrounding fluid.

The no-flow condition beyond the channel walls leads to a vanishing probability current at those boundaries. Consequently, due to the impenetrability of the 
channel walls, the normal component of the probability current $\boldsymbol{J}(\boldsymbol{x}, t)$ vanishes at those boundaries. Thus, the boundary conditions at the channel walls are given by

$$
\boldsymbol{J}(\boldsymbol{x}, t) \cdot \boldsymbol{n}=0, \quad \boldsymbol{x} \in \text { channel wall, }
$$

where $\boldsymbol{n}$ denotes the normal vector at the channel walls.

The boundary of a two-dimensional periodic channel, which is mirror symmetric about the $x$-axis, is given by the dimensionless periodic functions $y= \pm \omega(x)$, i.e. $\omega(x+1)=\omega(x)$ for all $x$, where $x$ and $y$ are the cartesian components of $\boldsymbol{x}$. In this case, the boundary condition reads

$$
\frac{\mathrm{d} \omega(x)}{\mathrm{d} x}\left[f P(x, y, t)-\frac{\partial P(x, y, t)}{\partial x}\right]+\frac{\partial P(x, y, t)}{\partial y}=0,
$$

at $y= \pm \omega(x)$. Except for a straight channel with $\omega=$ const., there are no periodic channel shapes for which an exact analytical solution of the Fokker-Planck equation $(2.6 a, 2.6 b)$ with the elaborate boundary conditions in equation $(2.8)$ is presently known. Approximate solutions though can be obtained on the basis of a one-dimensional diffusion problem proceeding in an effective potential. Narrow channel openings, which act as geometric hindrances in the original system, then manifest themselves as entropic barriers within an effective one-dimensional diffusive FJ approximation (Jacobs 1967; Zwanzig 1992; Reguera \& Rubi 2001; Kalinay \& Percus 2006; Reguera et al. 2006; Burada et al. 2007).

\section{Equilibration in transverse channel directions: the Fick-Jacobs approximation}

In the absence of an external force, i.e. for $\boldsymbol{f}=0$, it was shown (Jacobs 1967; Zwanzig 1992; Reguera \& Rubi 2001; Kalinay \& Percus 2006) that the dynamics of Brownian particles in confined structures (such as the one depicted in figure 1) can be described approximatively by the FJ equation, i.e.

$$
\frac{\partial}{\partial t} P(x, t)=\frac{\partial}{\partial x} D(x) \mathrm{e}^{-A(x)} \frac{\partial}{\partial x} \mathrm{e}^{A(x)} P(x, t) .
$$

This one-dimensional equation is obtained from the full two-dimensional Smoluchowski equation upon the elimination of the transverse $y$ spatial coordinate degree of freedom by assuming a much faster equilibration in that channel direction than in the longitudinal one. An analogous reduction mechanism has been used for the transport of neutrons through nuclear reactors (Beckurts \& Wirtz 1964). In equation (3.1), $P(x, t)=\int_{-\omega(x)}^{\omega(x)} P(x, y, t) \mathrm{d} y$ denotes the marginal probability density along the axis of the channel. $A(x)$ corresponds to the potential of mean force, which equals, for the considered situation, the free energy, i.e. $A(x)=E(x)-S(x)=0-\ln \omega(x)$. We note that for three-dimensional channels, an analogue approximate Fokker-Planck equation holds in which the function $\omega(x)$ is to be replaced by $\pi \omega^{2}(x)$ (i.e. the area of the corresponding cross-section). In the original work by Jacobs (1967), the one-dimensional diffusion coefficient $D(x)$ is constant and equals the bare diffusion constant 


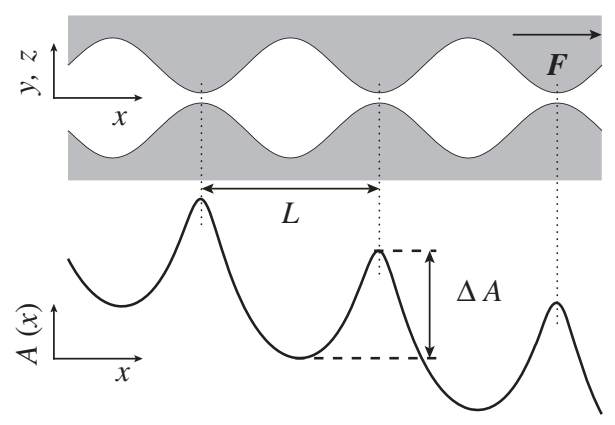

Figure 2. Sketch of the two-dimensional channel and the effective one-dimensional potential: the FJ approximation allows for a reduction of the two-dimensional Brownian dynamics within the periodic channel (periodicity $L$ ) to an approximate one-dimensional Brownian dynamics with an effective potential that is given by the free energy function $A(x)$. In the presence of an applied bias, $A(x)$ has the form of a tilted periodic potential with a barrier height of $\Delta A$ that depends on the temperature $T$.

that assumes unity in the present dimensionless variables. However, introducing the $x$-dependent diffusion coefficient considerably improves the accuracy of the kinetic equation, extending its validity to more winding structures (Zwanzig 1992; Reguera \& Rubi 2001; Burada et al. 2007). The expression for $D(x)$ reads (in dimensionless units)

$$
D(x)=\frac{1}{\left(1+\omega^{\prime}(x)^{2}\right)^{\alpha}},
$$

where $\alpha=1 / 3,1 / 2$ for two and three dimensions, respectively, has been shown to appropriately account for the curvature effects of the confining walls (Reguera \& Rubi 2001; Burada et al. 2008b) and $\omega^{\prime}(x)$ indicates the first derivative of the boundary function $\omega(x)$ with respect to $x$.

In the presence of a constant force $F$ along the direction of the channel, the FJ diffusion equation (3.1) can be recast into the form (Reguera et al. 2006; Burada et al. 2007, 2008b)

$$
\frac{\partial P}{\partial t}=\frac{\partial}{\partial x} D(x)\left(\frac{\partial P}{\partial x}+\frac{\mathrm{d} A(x)}{\mathrm{d} x} P\right)
$$

with the dimensionless free energy $A(x):=E(x)-S(x)=-f x-\ln \omega(x)$. In terms of the original unscaled physical variables, the energy is $\tilde{E} \equiv k_{\mathrm{B}} T E(x)=$ $-F \tilde{x}(\tilde{x}=x L)$ and the dimensional entropic contribution reads $\tilde{S} \equiv k_{\mathrm{B}} T S(x)=$ $k_{\mathrm{B}} T \ln \omega(x)$. For a periodic channel arrangement, this free energy assumes the form of a tilted periodic potential, see figure 2 . In the absence of a force, the free energy is purely entropic and equation (3.3) reduces to the FJ equation (3.1). On the other hand, for a straight channel, the entropic contribution vanishes and the particles are solely driven by the externally applied force.

Remarkably, the temperature $T$ dictates the strength of the effective potential. An increase in temperature causes an increase in barrier height $\Delta A$, while for purely energetic systems the barrier height is independent of the temperature (Hänggi et al. 1990). 


\section{(a) Steady-state probability density}

Formally, the steady-state density of the particles is obtained in the limit $t \rightarrow \infty$, i.e. $P^{\text {st }}(x)=\lim _{t \rightarrow \infty} P(x, t)$. As a consequence, $\partial / \partial t P^{\text {st }}(x)=0$. An expression for the steady-state density can be derived from equation (3.1), using arguments detailed in appendix A. Using the main result in equation (A 12), one obtains

$$
P^{\mathrm{st}}(x)=\frac{I(x, f)}{\int_{0}^{1} I(z, f) \mathrm{d} z},
$$

where

$$
I(x, f)=\mathrm{e}^{-A(x)} \int_{x}^{x+1} \frac{\mathrm{e}^{A\left(x^{\prime}\right)}}{D\left(x^{\prime}\right)} \mathrm{d} x^{\prime}
$$

depends on the dimensionless position $x$, the force $f$ and, via the positiondependent diffusion coefficient, on the shape of the tube given in terms of the shape function $\omega(x)$ and its first derivative, cf. equation (3.2). Note that the probability density $P^{\text {st }}(x)$ is normalized on the unit interval.

\section{(b) Nonlinear mobility}

The primary quantity of particle transport through periodic channels is the average particle current, $\langle\dot{x}\rangle$, or equivalently, the nonlinear mobility, which is defined as the ratio between the average particle current and the applied force $f$. For the average particle current, we derive an expression that is similar to the Stratonovich formula for the current occurring in titled periodic energy landscapes, but here, with a spatially dependent diffusion coefficient (Burada et al. 2007). A detailed derivation of this expression is given in appendix A, cf. equation (A 11). Hence, we obtain the nonlinear mobility for a two- or three-dimensional channel

$$
\mu(f)=\frac{\langle\dot{x}\rangle}{f}=\frac{1}{f} \frac{1-\mathrm{e}^{-f}}{\int_{0}^{1} I(z, f) \mathrm{d} z},
$$

with $I(z, f)$ given in equation $(3.5)$.

\section{Precise numerics for a two-dimensional channel geometry}

The steady-state density and the average particle current, predicted analytically earlier, has been compared with Brownian dynamic simulations performed by a numerical integration of the Langevin equation (2.4), using the stochastic Euler algorithm. The shape of the exemplarily taken two-dimensional channel is described by

$$
\omega(x):=a \sin (2 \pi x)+b,
$$

where $b>a$. The sum and difference of the two parameters $a+b$ and $b-a$ yield half of the maximal and the minimal width of the channel, respectively. Moreover, a controls the slope of the channel walls, which, in turn, determines the one-dimensional diffusion coefficient $D(x)$.

For the considered channel configuration, cf. equation (4.1), the boundary condition becomes $\omega(x)=a[\sin (2 \pi x)+\kappa]$, where $\kappa=b / a=1.02$ throughout this paper. For $a$ we chose values between 1 and $1 / 2 \pi$. In all cases, the width of the 


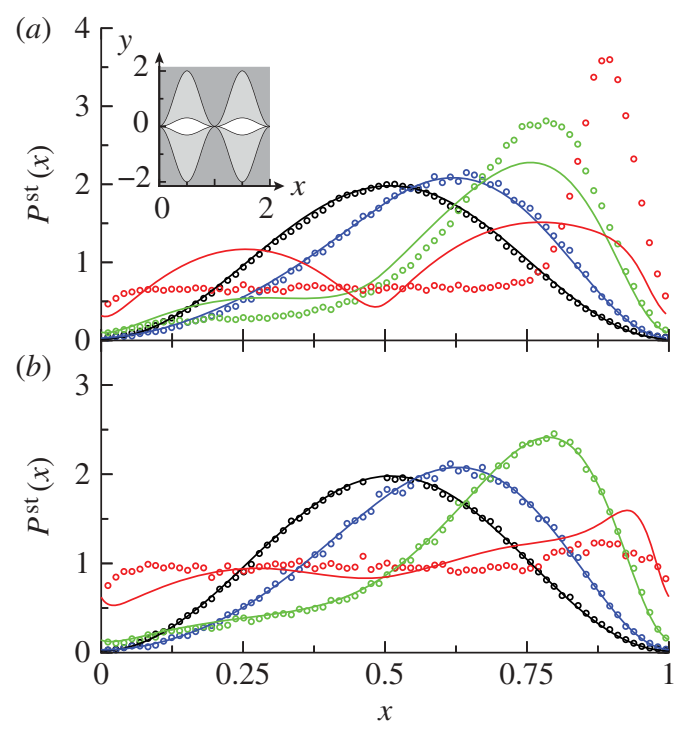

Figure 3. The normalized steady-state probability density of particles along the propagation direction is depicted for different force values $f$ (black, $f=0.2$; blue, $f=3.0$; green, $f=10.0$; red, $f=50.0$ ) for two different two-dimensional channels (see inset in panel $(a)$ ), with the scaled halfwidth shape function given by $\omega(x)=a[\sin (2 \pi(x-0.25))+1.02]$ (the shift -0.25 ensures that the bottlenecks are located at 0 and 1 ). ( $a$ ) For $a=1$, the maximal and minimal channel widths are 4.04 and 0.04 , respectively, and $(b)$ for $a=1 / 2 \pi$, they are $6.43 \times 10^{-1}$ and $6.37 \times 10^{-3}$, respectively. The solid lines correspond to the steady-state probability density obtained from the one-dimensional FJ approximation, equations (3.4) and (3.5), and symbols correspond to two-dimensional numerical simulations, see equation (4.2).

widest opening within the channel is larger by a factor of 100 than the width at the narrowest opening. One may therefore expect rather strong entropic effects for these channel geometries.

\section{(a) Stationary probability densities}

We have evaluated the stationary probability density $P^{\text {st }}(x, y)$, in the long time limit, by mapping all particle positions onto the primitive cell by translation into the longitudinal channel direction. Consequently, $\int_{0}^{1} \mathrm{~d} x \int_{-\omega(x)}^{\omega(x)} \mathrm{d} y P^{\mathrm{st}}(x, y)=1$. Figure 3 (solid lines) depicts the normalized steadystate probability density in the $x$-direction for various scaling parameter values, derived from the reduced one-dimensional FJ result, equation (A 12), and is compared with the numerical simulations for the exact expression

$$
P^{\mathrm{st}}(x):=\frac{\int_{-\omega(x)}^{\omega(x)} P^{\mathrm{st}}(x, y) \mathrm{d} y}{\int_{0}^{1} \mathrm{~d} x \int_{-\omega(x)}^{\omega(x)} P^{\mathrm{st}}(x, y) \mathrm{d} y} .
$$

Note that the steady-state marginal density $P^{\text {st }}(x)$ is normalized on the primitive cell.

At small scaling parameter values $f$, the one-dimensional steady-state density given by equation (3.4) is in very good agreement with those obtained from numerical simulations, see figure 3 . This holds true for rather arbitrary channel 
geometry (not shown). However, the comparison fails for large $f$ values of the scaling parameter or for more winding structures corresponding to larger $a$ values. When increasing the force, the maximum of $P^{\text {st }}(x)$ is shifted towards the exit of the cell, and the particles mostly accumulate in front of the bottleneck, see figure $3 a$, and the one-dimensional kinetic description starts to fail in that forward bottleneck $x$-region. However, by decreasing the $a$ of the geometric channel shape function, the accuracy of the FJ approximation considerably improves up to very large force values $f$, see figure $3 b$.

As a common feature, one observes that, for the two chosen geometric structures, in the large-force regime the numerically obtained $P^{\text {st }}(x)$ is essentially constant over a wide range of $x$ values, indicating a minor influence of the shape of the structure on the dynamics of the laterally forward-forced particles. In this situation, the thermal noise plays a minor role and the deterministic dynamics (with diffusion set to zero) of the diffusive equation provides a good starting point. Put differently, at strong longitudinal driving strength the correction in the diffusion coefficient leading to a spatial dependency, i.e. $D(x)$, overestimates the role of the entropic effects and consequently the FJ approximation starts failing over extended $x$-regimes.

The reasons for the failure of the FJ approximation for large forces become obvious when checking the equilibration assumption in the transverse channel direction. From our simulations, we can actually analyse the validity of the hypotheses of equilibration in the transverse direction on which the FJ description relies. A detailed analysis is provided by testing the normalized steady-state probability density in the transverse direction at a given $x$-position, i.e.

$$
P_{x}^{\mathrm{st}}(y):=\frac{P^{\mathrm{st}}(x, y)}{\int_{-\omega(x)}^{\omega(x)} P^{\mathrm{st}}(x, y) \mathrm{d} y} .
$$

In figure 4, we depict the steady-state probability density at the position of maximal channel width. For small values of the scaling parameter $f$, the $P_{x}^{\text {st }}(y)$ is very flat, indicating an almost ideal homogeneous equilibration in the transverse direction, as required by the FJ approximation scheme. However, at large force strengths $f$, the Brownian particles concentrate along the axis of the channel with $y=0$. In this situation, the assumption of equilibration along the transverse direction fails, and the density peaks around the $y=0$ value. The particles can only feel the presence of the boundaries when they are close to the bottlenecks. Hence, in the limit of very large force values, the influence of the entropic barriers practically disappears.

\section{(b) Nonlinear mobility}

The average particle current was derived from an ensemble average using $3 \times 10^{4}$ trajectories

$$
\langle\dot{x}\rangle=\lim _{t \rightarrow \infty} \frac{\langle x(t)\rangle}{t} .
$$

Figure 5 shows the nonlinear mobility as a function of the scaling parameter $f$. We note that the transport in one-dimensional periodic energetic potentials distinctly differs from the one occurring in one-dimensional periodic systems 
in the presence of entropic barriers (Reguera et al. 2006). The fundamental difference lies in the temperature dependence of these barrier shapes. Decreasing the temperature in an energetic periodic potential decreases the transition rates from one cell to the neighbouring one by decreasing the Arrhenius factor $\exp \left\{-\Delta V /\left(k_{\mathrm{B}} T\right)\right\}$, where $\Delta V$ denotes the activation energy necessary to proceed to a period (Hänggi et al. 1990). Hence, decreasing the temperature yields a decreasing nonlinear mobility. For a one-dimensional periodic system with an entropic free energy (or entropic potential of mean force), a decrease of temperature results, however, in an increase of the dimensionless force parameter $f$ and consequently, in a monotonic increase of the nonlinear mobility, cf. figure 5 .

The dependence of the dynamics on the geometry parameter $a$ nicely reflects the entropic effects on the mobility: a channel with a larger $a$ value has wider openings, and thus provides more configuration space where the particle can sojourn. This longer residence time within a period of the channel diminishes the throughput and consequently the mobility. This is corroborated by the results of our calculations depicted in figure 5 . For all values of $f$, an increase in value of $a$ leads to a decrease in the mobility. This holds not only in regimes for which the FJ equation is valid, but also for large values of $f$ where the approximation fails. For very large values of the scaling parameter $f$, the nonlinear mobility approaches the value 1, i.e. it agrees with the deterministic strong driving limit.

By means of the nonlinear mobility, a detailed comparison between twodimensional simulation results and the analytic results, cf. equation (3.6), enables one to determine validity criteria for the FJ approximation, for further details see Burada et al. (2007, 2008b).

\section{Effective diffusion and the Sutherland-Einstein relation}

A validity of a nonlinear Sutherland-Einstein relation implies that, in physical units, we can relate the nonlinear mobility $\mu(F)$ directly to the nonlinear, effective $x$-diffusion $D_{\text {eff }}(F)$, reading

$$
D_{\text {eff }}(F)=\mu(F) k_{\mathrm{B}} T .
$$

Put differently, the effective diffusion coefficient $D_{\text {eff }}$ for the diffusive spreading along the longitudinal channel direction would then solely be determined by the nonlinear mobility discussed earlier and the environmental temperature $T$.

A validity of this relation would then imply a monotonic increase towards the entropic-free diffusion limit, i.e. $D_{\text {eff }}=k_{\mathrm{B}} T / \eta$. The latter is being approached in the strong forcing limit, where entropic effects cease to play a significant role. Such a monotonic behaviour, however, is not observed from the numerical simulations for the effective $x$-diffusion coefficient (Reguera et al. 2006; Burada et al. 2008b). It is defined as the ratio between the asymptotic behaviour of the variance of the position variable and the elapsed time $t$, i.e.

$$
D_{\text {eff }}=\lim _{t \rightarrow \infty} \frac{\left\langle x^{2}(t)\right\rangle-\langle x(t)\rangle^{2}}{2 t} .
$$




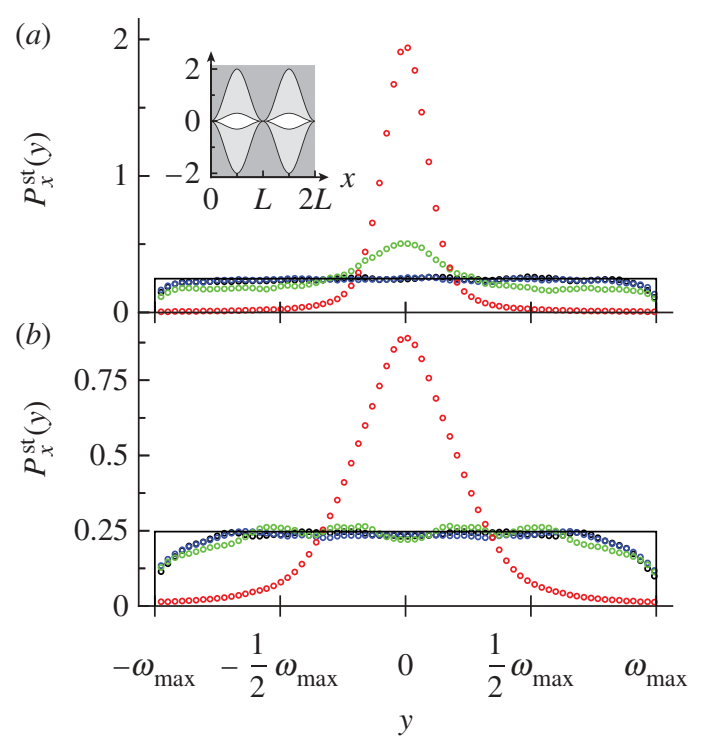

Figure 4. The normalized steady-state probability density of particles in the $y$-direction $P_{x}^{\text {st }}(y)$, cf. equation (4.3), taken at the $x$-position of maximal channel width, is depicted for different values of the scaling parameter $f$ (black circles, $f=0.2$; blue circles, $f=3.0$; green circles, $f=10.0$; red circles, $f=50.0$ ) and different channel geometries (see inset in panel $(a)$ ) for the boundary function $\omega(x)=$ $a[\sin (2 \pi(x-0.25))+1.02]$ (the shift -0.25 ensures that the bottlenecks are located at 0 and 1$)$. The maximal channel widths for the two structures are (a) $2 \omega_{\max }=4.04$ for $a=1$ and (b) $2 \omega_{\max }=$ $6.43 \times 10^{-1}$ for $a=1 / 2 \pi$. The symbols correspond to two-dimensional numerical simulations, see equation (4.3). For large scaled force values $f$, the numerically obtained steady-state densities deviate from the uniform, i.e. box-like, density (shown by the black solid line). This indicates the failure of the equilibration assumption on which the FJ approximation relies.

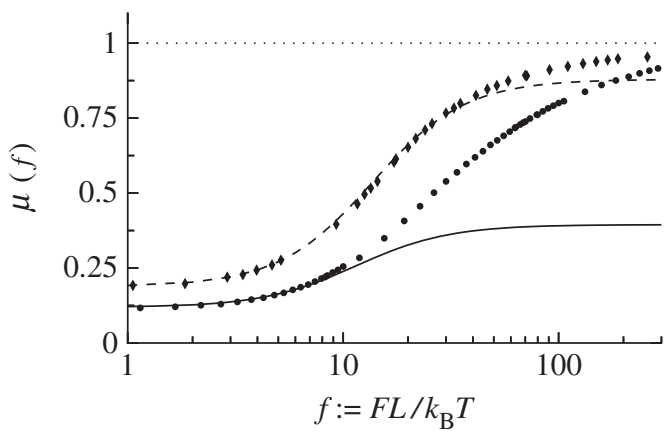

Figure 5. The numerically simulated (symbols) and analytically calculated (cf. equation (3.6); lines) dependence of the scaled nonlinear mobility $\mu(f)$ versus the dimensionless force $f=F L / k_{\mathrm{B}} T$ is depicted for two two-dimensional channel geometries. For both channels, the scaled half-width is given by $\omega(x)=a[\sin (2 \pi x)+1.02] ; a=1$, circles and solid line; $a=1 /(2 \pi)$, diamonds and dashed line. The dotted line indicates the deterministic limit $\mu(f)=\langle\dot{x}\rangle / f=1$. 


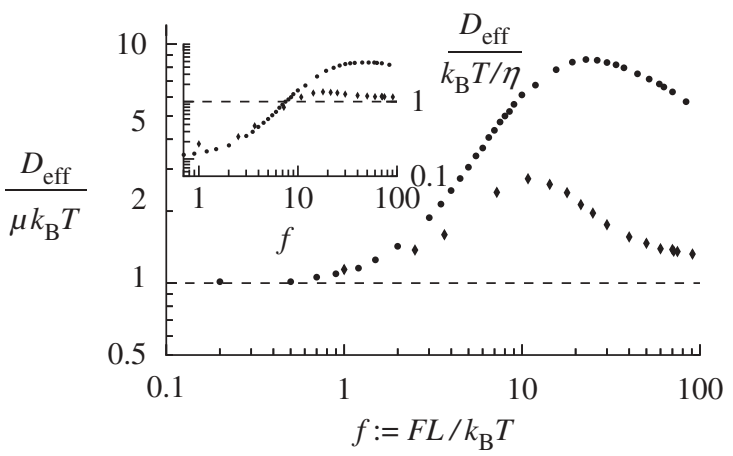

Figure 6 . The ratio of the effective diffusion $D_{\text {eff }}$ and nonlinear mobility $\mu$ times the thermal energy $k_{\mathrm{B}} T$ is depicted as a function of the scaling parameter $f$ for two channel geometries: $\omega(x)=a[\sin (2 \pi x)+1.02]$ with $a=1$ (circles) and $a=1 / 2 \pi$ (diamonds). The dashed horizontal line at 1 indicates the validity of the Sutherland-Einstein relation in this non-equilibrium situation $D_{\text {eff }} /\left(\mu k_{\mathrm{B}} T\right)=1$. A deviation from this line consequently marks the breakdown of this relationship. The inset depicts the ratio of the effective diffusion $D_{\text {eff }}$ and bulk diffusion constant, being $k_{\mathrm{B}} T / \eta$. A ratio larger than 1 (dashed horizontal line) indicates a characteristic enhancement of the effective $x$-diffusion.

Interestingly, the dependence of the effective diffusion coefficient on the scaling parameter exhibits a bell-shaped behaviour, cf. inset of figure 6 , thus indicating a failure of the Sutherland-Einstein relation in this moderate-to-strong driving regime. This breakdown of the Sutherland-Einstein relation can also be detected within the FJ description (not shown in figure 6). The FJ approximation for this effective $x$-diffusion also yields a non-monotonic dependence of the effective $x$-diffusion coefficient on the scaling parameter $f$, exhibiting a peak value exceeding the bulk diffusion coefficient $D_{0}=k_{\mathrm{B}} T / \eta$ (Reguera et al. 2006; Burada et al. $2008 b)$.

For a detailed comparison, we depict the ratio of the numerically obtained $D_{\text {eff }}$ and $\left(\mu k_{\mathrm{B}} T\right)$ in figure 6 . Surprisingly, it turns out that such a SutherlandEinstein relation, equation (5.1), holds true in terms of the effective mobility in the small forcing limit $F \rightarrow 0$, i.e. in the linear response regime. It increasingly fails, however, for increasing bias strength $F$. At very strong bias, i.e. $F \rightarrow \infty$, the biased diffusion becomes effectively 'free' from entropic effects and expectedly approaches the free limit, given by $k_{\mathrm{B}} T / \eta$, which renders the original, linear Sutherland-Einstein result in terms of the $F$-independent mobility $\mu=1 / \eta$. Put differently, the influence of entropic barriers caused by the bottlenecks becomes negligible at strong bias. Vice versa, the bell-shaped behaviour of the ratio depicted in figure 6 reflects the fact that this effective diffusion is not increasing monotonically but rather, exhibits an enhancement of effective diffusion at moderate bias (or scaling) values $F$, cf. in the inset of figure 6 .

\section{Conclusions}

In summary, we demonstrated the applicability of the equilibration approximation in describing biased diffusive transport occurring in narrow, irregularly shaped one-dimensional channel structures. The FJ description, which relies on the 
equilibration assumption, allows for a treatment of the dynamics within an effective one-dimensional kinetic equation of the Smoluchowski form. Bottlenecks and other confining restrictions of available configuration space yield, within this approximation, an effective one-dimensional diffusion equation exhibiting entropic barriers. Owing to the intrinsic temperature dependence of the underlying entropic free energy contribution, one finds the transport phenomena in periodic channels possessing varying cross section features that are radically different from conventional transport occurring in energetic periodic potential landscapes.

The most striking difference between these two physical situations is that, for a fixed channel geometry, the dynamics is characterized by a single scaling parameter $f=F L /\left(k_{\mathrm{B}} T\right)$ which combines the external force $F$ causing a drift, the period length $L$ of the channel, and the thermal energy $k_{\mathrm{B}} T$. The latter presents a measure of the strength of the acting fluctuating thermal forces. This leads to an opposite temperature dependence of the mobility. While the mobility of a particle in an energetic potential increases with increasing temperature, the mobility of a particle undergoing biased diffusion in an irregular channel decreases. The incorporation of the spatial variation of the channel width in terms of an entropic free energy contribution allows for a quantitative understanding of the dependence of the transport properties, such as the nonlinear mobility, on parameters such as force strength, channel topology or temperature. Moreover, the lateral steady-state probability densities $P^{\mathrm{st}}(x)$ can be evaluated in analytical closed form within the reduced kinetic FJ approximation, see appendix A.

Such an effective one-dimensional reduction of a complex diffusion dynamics with intricate boundary conditions at the confining walls certainly proves useful and beneficial for the quantitative description, design and control of diffusive transport along tortuous pores and the like. The latter situation dictates the stochastic far-from-equilibrium transport in a great variety of biological and structured synthetic pores and confining cavities, such as buckyballs, zeolites, etc. As an example, this FJ approximation has successfully been used in describing the phenomenon of stochastic resonance (Gammaitoni et al. 1998; Hänggi 2002) in a two-dimensional system exhibiting an entropic barrier (Burada et al. 2008a).

This work has been supported by the Volkswagen Foundation (project I/80424, P.H.) the DFG via research center, SFB-486, project A10 (G.S., P.H.) and via the DFG project no. 1517/26-1 (P.S.B., P.H.) and by the German Excellence Initiative via the Nanosystems Initiative Munich (P.H., P.S.B.).

\section{Appendix A. Steady-state current and probability density}

In this appendix, we derive the steady-state solution for the effective, onedimensional dimensionless FJ (Smoluchowski-type) equation, equation (3.1),

$$
\frac{\partial}{\partial t} P(x, t)=\frac{\partial}{\partial x} D(x) \mathrm{e}^{-A(x)} \frac{\partial}{\partial x} \mathrm{e}^{A(x)} P(x, t),
$$

where $A(x)$ denotes the free energy function, $A(x)=-f x-\ln \omega(x)$, with $\omega(x+1)=\omega(x)$. Equation (A 1) results from the probability continuity equation

$$
\frac{\partial}{\partial t} P(x, t)=-\frac{\partial}{\partial x} J(x, t),
$$


with the probability current $J(x, t)$ reading

$$
J(x, t)=-D(x) \mathrm{e}^{-A(x)} \frac{\partial}{\partial x} \mathrm{e}^{A(x)} P(x, t) .
$$

In the case of a tilted periodic potential, i.e. $A(x+1)=A(x)-f$, it is convenient to define the reduced probability density and the corresponding reduced current, i.e.

$$
\hat{P}(x, t)=\sum_{n} P(n+x, t)
$$

and

$$
\hat{J}(x, t)=\sum_{n} J(n+x, t), \quad n \in \mathbb{Z}
$$

By definition, these functions are periodic with periodicity $L=1, \hat{P}(x+1, t)=$ $\hat{P}(x, t), \hat{J}(x+1, t)=\hat{J}(x, t)$. The same holds true for the spatially dependent diffusion coefficient, i.e. $D(x+1)=D(x)$. Moreover, $\hat{P}(x, t)$ and $\hat{J}(x, t)$ obey the continuity equation, equation (A 2$)$, and $\hat{P}(x, t)$ is normalized on an interval $(x, x+1)$, provided that $P(x, t)$ is normalized, i.e. $\int_{-\infty}^{+\infty} P(x, t) \mathrm{d} x=1$.

In the steady-state limit, the probability current assumes a constant, i.e. $\hat{J}(x, t) \rightarrow \hat{J}$. Thus, equation (A 3$)$ becomes

$$
\hat{J}=-D(x) \mathrm{e}^{-A(x)} \frac{\partial}{\partial x} \mathrm{e}^{A(x)} \hat{P}^{\mathrm{st}}(x) .
$$

Multiplying both sides of equation (A 6$)$ by $\mathrm{e}^{A(x)} / D(x)$, and integrating over a period $L=1$, we obtain

$$
\hat{J} \int_{x}^{x+1} \frac{\mathrm{e}^{A\left(x^{\prime}\right)}}{D\left(x^{\prime}\right)} \mathrm{d} x^{\prime}=-\int_{x}^{x+1} \frac{\partial}{\partial x^{\prime}} \mathrm{e}^{A\left(x^{\prime}\right)} \hat{P}^{\mathrm{st}}\left(x^{\prime}\right) \mathrm{d} x^{\prime},
$$

which simplifies to

$$
\hat{J} \int_{x}^{x+1} \frac{\mathrm{e}^{A\left(x^{\prime}\right)}}{D\left(x^{\prime}\right)} \mathrm{d} x^{\prime}=\hat{P}^{\mathrm{st}}(x)\left(1-\mathrm{e}^{-f}\right) \mathrm{e}^{A(x)} .
$$

Upon rearranging the terms on the right-hand side and integrating once more over a period, i.e. from 0 to 1 , we find the first result

$$
\hat{J}=\frac{\left(1-\mathrm{e}^{-f}\right)}{\int_{0}^{1} \mathrm{e}^{-A(x)} \mathrm{d} x \int_{x}^{x+1} \frac{\mathrm{e}^{A\left(x^{\prime}\right)}}{D\left(x^{\prime}\right)} \mathrm{d} x^{\prime}} .
$$


Here, we made use of the normalization condition of the stationary probability, i.e. $\int_{0}^{1} \hat{P}^{\mathrm{st}}(x) \mathrm{d} x=1$. The general relation between the steady-state probability current and the steady-state average particle current $\langle\dot{x}\rangle$ is

$$
\langle\dot{x}\rangle=\int_{0}^{1} \hat{J} \mathrm{~d} x,
$$

which implies that $\langle\dot{x}\rangle=\hat{J}$. Thus, the transport current is given by the first main result

$$
\langle\dot{x}\rangle=\frac{\left(1-\mathrm{e}^{-f}\right)}{\int_{0}^{1} \mathrm{e}^{-A(x)} \mathrm{d} x \int_{x}^{x+1} \frac{\mathrm{e}^{A\left(x^{\prime}\right)}}{D\left(x^{\prime}\right)} \mathrm{d} x^{\prime}} .
$$

By substituting equation (A 9) back into equation (A 8), we obtain, for the steady-state probability density in the $x$-direction, the second main result

$$
\hat{P}^{\mathrm{st}}(x)=\frac{\mathrm{e}^{-A(x)} \int_{x}^{x+1} \frac{\mathrm{e}^{A\left(x^{\prime}\right)}}{D\left(x^{\prime}\right)} \mathrm{d} x^{\prime}}{\int_{0}^{1} \mathrm{e}^{-A(x)} \mathrm{d} x \int_{x}^{x+1} \frac{\mathrm{e}^{A\left(x^{\prime}\right)}}{D\left(x^{\prime}\right)} \mathrm{d} x^{\prime}} .
$$

\section{References}

Astumian, R. D. \& Hänggi, P. 2002 Brownian motors. Phys. Today 55, 33-39. (doi:10.1063/ $1.1535005)$

Barrer, R. M. 1978 Zeolites and clay minerals as sorbents and molecular sieves. London, UK: Academic Press

Beckurts, K. H. \& Wirtz, K. 1964 Neutron physics. Berlin, Germany: Springer.

Berezhkovskii, A. M. \& Bezrukov, S. M. 2005 Optimizing transport of metabolites through large channels: molecular sieves with and without binding. Biophys. J. 88, L17-L19. (doi:10.1529/biophysj.104.057588)

Burada, P. S., Schmid, G., Reguera, D., Rubí, J. M. \& Hänggi, P. 2007 Biased diffusion in confined media: test of the Fick-Jacobs approximation and validity criteria. Phys. Rev. E 75, 051111. (doi:10.1103/PhysRevE.75.051111)

Burada, P. S., Schmid, G., Reguera, D., Vainstein, M. H., Rubí, J. M. \& Hänggi, P. $2008 a$ Entropic stochastic resonance. Phys. Rev. Lett. 101 130602. (doi:10.1103/PhysRevLett. 101.130602)

Burada, P. S., Schmid, G., Talkner, P., Hänggi, P., Reguera, D. \& Rubí, J. M. $2008 b$ Entropic particle transport in periodic channels. Biosyst. 93, 16-22. (doi:10.1016/ j.biosystems.2008.03.006)

Burada, P. S., Hänggi, P., Marchesoni, F., Schmid, G. \& Talkner, P. 2009 Diffusion in confined geometries. Chem. Phys. Chem. 10, 45-54. (doi:10.1002/cphc.200800526)

Derenyi, I. \& Astumian, R. D. 1998 AC separation of particles by biased Brownian motion in a two-dimensional sieve. Phys. Rev. E 58, 7781-7784. (doi:10.1103/PhysRevE.58.7781)

Gammaitoni, L., Hänggi, P., Jung, P. \& Marchesoni, F. 1998 Stochastic resonance. Rev. Mod. Phys. 70, 223-288. (doi:10.1103/RevModPhys.70.223)

Hänggi, P. 2002 Stochastic resonance in biology. Chem. Phys. Chem. 3, 285-290. (doi:10.1002/1439-7641(20020315)3:3<285::AID-CPHC285>3.0.CO;2-A)

Hänggi, P. \& Marchesoni, F. 2005 Introduction: 100 years of Brownian motion. Chaos 15, 026101. (doi:10.1063/1.1895505)

Hänggi, P. \& Marchesoni, F. 2009 Artificial Brownian motors: controlling transport on the nanoscale. Rev. Mod. Phys. 81, 387-442. (doi:10.1103/RevModPhys.81.387)

Hänggi, P. \& Thomas, H. 1982 Stochastic processes: time-evolution, symmetries and linear response. Phys. Rep. 88, 207-319. (doi:10.1016/0370-1573(82)90045-X) 
Hänggi, P., Talkner, P. \& Borkovec, M. 1990 Reaction rate theory: fifty years after Kramers. Rev. Mod. Phys. 62, 251-342. (doi:10.1103/RevModPhys.62.251)

Hille, B. 2001 Ion channels of excitable membranes. Sunderland, MA: Sinauer.

Jacobs, M. H. 1967 Diffusion processes. New York, NY: Springer.

Kalinay, P. \& Percus, J. K. 2006 Corrections to the Fick-Jacobs equation. Phys. Rev. E 74, 041 203. (doi:10.1103/PhysRevE.74.041203)

Kettner, C., Reimann, P., Hänggi, P. \& Müller, F. 2000 Drift ratchet. Phys. Rev. E 61, 312-323. (doi:10.1103/PhysRevE.61.312)

Kosinska, I. D., Goychuk, I., Kostur, M., Schmid, G. \& Hänggi, P. 2008 Rectification in synthetic conical nanopores: a one-dimensional Poisson-Nernst-Planck modeling. Phys. Rev. E 77, 031 131. (doi:10.1103/PhysRevE.77.031131)

Liu, L., Li, P. \& Asher, S. A. 1999 Entropic trapping of macromolecules by mesoscopic periodic voids in a polymer hydrogel. Nature 397, 141-144. (doi:10.1038/16426)

Matthias, S. \& Müller F. 2003 Asymmetric pores in a silicon membrane acting as massively parallel Brownian ratchets. Nature 424, 53-57. (doi:10.1038/nature01736)

Mazo, R. M. 2002 Brownian motion: fluctuations, dynamics and applications. Oxford, UK: Clarendon Press.

Müller, F., Birner, A., Schilling J., Gösele, U., Kettner, C. \& Hänggi, P. 2000 Membranes for micropumps from macroporous silicon. Phys. Stat. Sol. A 182, 585-590. (doi:10.1002/1521396X(200011) 182:1<585::AID-PSSA585>3.0.CO;2-4)

Nixon, G. I. \& Slater, G. W. 2002 Saturation and entropic trapping of monodisperse polymers in porous media. J. Chem. Phys. 117, 4042-4046. (doi:10.1063/1.1493189)

Purcell, E. M. 1977 Life at low Reynolds number. Am. J. Phys. 45, 3-11. (doi:10.1119/1.10903)

Reguera, D. \& Rubí, J. M. 2001 Kinetic equations for diffusion in the presence of entropic barriers. Phys. Rev. E 64, 061 106. (doi:10.1103/PhysRevE.64.061106)

Reguera, D., Schmid, G., Burada, P. S., Rubí, J. M., Reimann, P. \& Hänggi, P. 2006 Entropic transport: kinetics, scaling and control mechanisms. Phys. Rev. Lett., 96, 130603. (doi:10.1103/PhysRevLett.96.130603)

Reimann, P. \& Hänggi, P. 2002 Introduction to the physics of Brownian motors. Appl. Phys. A 75, 169-178. (doi:10.1007/s003390201331)

Risken, H. 1989 The Fokker-Planck equation. Berlin, Germany: Springer.

Siwy, Z., Kosinska, I. D., Fulinski, A. \& Martin, C. R. 2005 Asymmetric diffusion through synthetic nanopores. Phys. Rev. Lett. 94, 048 102. (doi:10.1103/PhysRevLett.94.048102)

Volkmuth, W. D. \& Austin, R. H. 1992 DNA electrophoresis in microlithographic arrays. Nature 358, 600-602. (doi:10.1038/358600a0)

Zwanzig, R. 1992 Diffusion past an entropic barrier. J. Phys. Chem. 96, 3926-3920. (doi:10.1021/ j100189a004) 